\title{
Measuring Lateral Diffusion of Receptors On Plasma Membrane of Macrophages Using Raster Image Correlation Spectroscopy
}

\author{
S. Makaremi ${ }^{1}$, S. Ranjit ${ }^{2}$, M.A. Digman ${ }^{2}$, E. Gratton ${ }^{2}$, D. M.E. Bowdish ${ }^{3,4}$ and J.M. Moran-Mirabal ${ }^{1,5}$ \\ 1. School of Biomedical Engineering, McMaster University, ON, Canada. \\ 2. Biomedical Engineering, University of California Irvine, Irvine, CA. \\ ${ }^{3 .}$ Department of Pathology and Molecular Medicine, McMaster University, ON, Canada. \\ 4. MG DeGroote Institute for Infectious Diseases, McMaster University, ON, Canada. \\ ${ }^{5}$. Department of Chemistry \& Chemical Biology, McMaster University, ON Canada.
}

Macrophages are the sentinel cells of the innate immune system and play a pivotal role in the elimination of pathogens such as bacteria, cellular debris, and foreign entities. Macrophages express several plasma membrane receptors including Toll-like receptors (TLRs) and scavenger receptors which participate in the inflammatory responses accompanied by detection of pathogens and microbial internalization and ingestion, a process known as phagocytosis [1].

The spatial distribution of immunoreceptors and their lateral diffusion in the plasma membrane impact receptor-mediated signaling. Lateral clustering of receptors is essential for their activation as increased local density of receptors enhances the efficiency of signal transduction whenever cooperativity between multiple molecular players is required. The barriers to molecular diffusion imposed by the cytoskeleton lead to clustering of receptors and nanodomain formation. Also, the interactions of receptors with membrane components such as lipid rafts and cholesterol mediate their lateral diffusion in the plasma membrane [2].

While previous studies on immunoreceptors have provided important new insights into macrophages' behaviour and their interactions with pathogens, there is a lack of information about diffusion of specific membrane receptors and their role in inflammatory responses and phagocytic function.

In this study, we have investigated the lateral diffusion of a number of receptors including TLR2 in the plasma membrane of bone marrow derived macrophages (Figure 1) and the macrophage cell line RAW264.7. Our experimental methodology includes Raster-scan Image Correlation Spectroscopy (RICS) for imaging live cells. The RICS technique has successfully been utilized in previous studies to measure the diffusion coefficient of proteins in live cells [3]. RICS allows us to measure the diffusion of membrane receptors in cells by separating the slow and immobile fraction of moving particles. Also, it is possible to spatially map the diffusion of proteins in different sections of the membrane, giving us a snapshot of the local dynamics for these species. We acquire multiple raster-scanned frames of each cell using confocal microscopy, and perform the RICS analysis with SimFCS software to measure the diffusion coefficient of receptors on macrophages (Figure 2).

The diffusion coefficient obtained from different regions in each of the basal and apical membranes varies significantly both for RAW264.7 and primary macrohpages. We believe this variation is due to the nonhomogenous nature of the plasma membrane. Also, our results show that the diffusion of TLR2 receptors on the basal membrane of macrophages are significantly slower compared with their counterparts on the apical membrane. 
To our knowledge, this is the first study that compares the lateral diffusion of receptors through different parts of macrophages' membranes. We believe the results of this investigation will open up new avenues for future therapeutic studies in immunology.

\section{References:}

[1] D. M. Underbill and B. Gantner, Microbes \& Infection. 6 (2004), p. 1368.

[2] S. Mueller et al, Biophysical Journal 101 (2011), p. 1651.

[3] M.A. Digman and E. Gratton, Microscopy Research \& Technique. 72 (2009), p. 323.

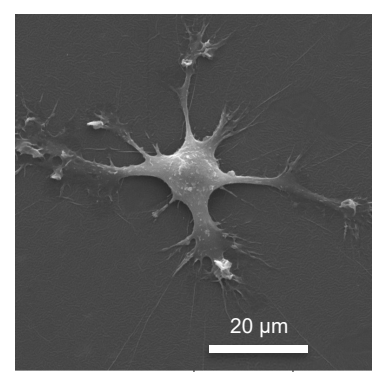

Figure 1. Scanning Electron Microscopy (SEM) image of a bone marrow derived macrophage.

(A)

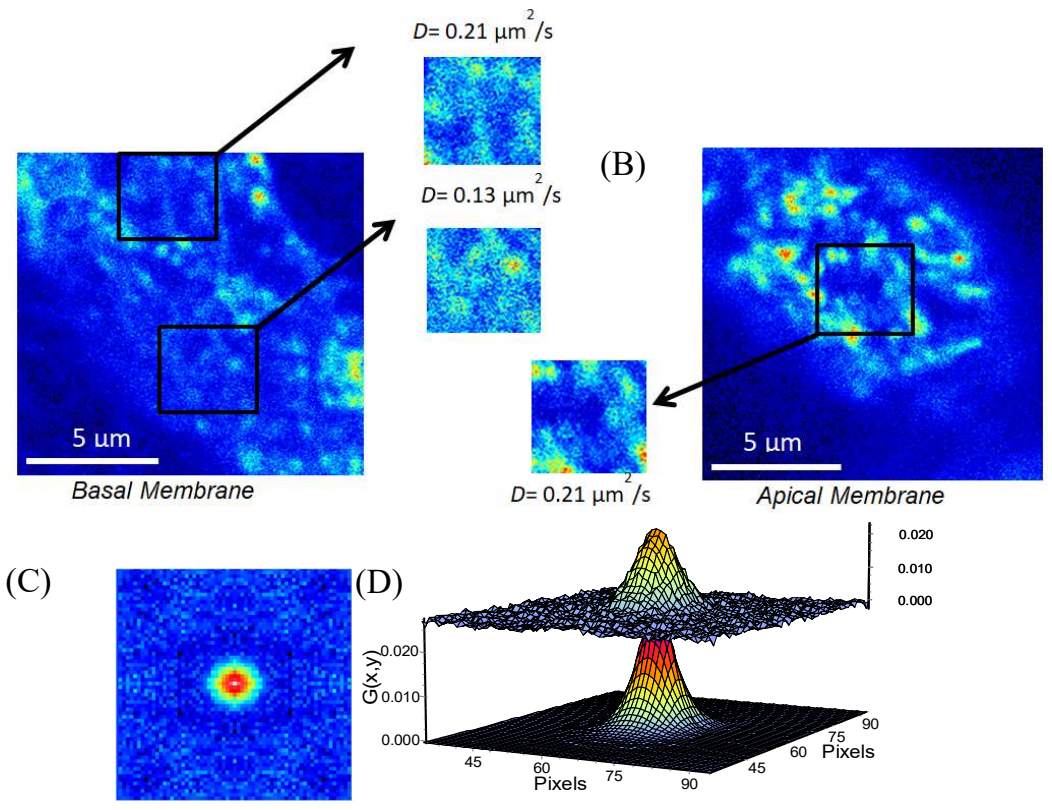

Figure 2. Example of RICS measurements on (A) basal and (B) apical membranes of a macrophage; (C) 2D representation of RICS autocorrelation function, (D) Plot of the data (upper surface) and 3D representation of the fit to the function (lower surface). 